\title{
Die Max Weber-Gesamtausgabe. Voreditionen - Konzeption der Ausgabe - Wichtige Ergebnisse
}

\author{
Wolfgang Schluchter
}

Angenommen: 4. März 2021 / Online publiziert: 30. März 2021

(C) Der/die Autor(en) 2021

\section{Vorbemerkung}

Nachdem Karl Marx am 14. März 1883 in London gestorben war, fand man in seinem Nachlass eine Fülle von begonnenen, aber nicht abgeschlossenen Manuskripten, darunter die Fortsetzung des Buches Das Kapital, dessen Band 1 er 1867 veröffentlicht hatte. Es war Friedrich Engels, der die weit gediehenen Bände 2 und 3 bearbeitete und schließlich 1885 bzw. 1894 veröffentlichte. Er folgte dabei der von Marx vorgesehenen Disposition, musste aber auch die eine oder andere Lücke schließen. Er tat es aus seiner intimen Kenntnis des Freundes heraus, mit dem er etwa 40 Jahre menschlich, wissenschaftlich und politisch eng verbunden gewesen war. Engels galt selbst als bedeutender Wissenschaftler, mit dem Verstorbenen ebenbürtig. Dennoch fiel ihm die Editionstätigkeit für den Freund nicht immer leicht. So berichtet er im Vorwort zum zweiten Band des Kapitals, der den Zirkulationsprozess behandelt, wie mühsam es war, das über einen längeren Zeitraum entstandene und immer wieder überarbeitete Manuskript in eine angemessene Form zu bringen. Er schreibt: „Die Hauptmasse des Materials war, wenn auch größtenteils sachlich, so doch nicht sprachlich fertig ausgearbeitet; abgefaßt in der Sprache, worin Marx seine Auszüge anzufertigen pflegte: nachlässiger Stil, familiäre, oft derbhumoristische Ausdrücke und Wendungen, englische und französische technische Bezeichnungen, oft ganze Sätze und selbst Seiten englisch; es ist Niederschrift der Gedanken in der Form, wie sie sich jedesmal im Kopf des Verfassers entwickelten. Neben einzelnen, ausführlich dargestellten Partien andre, gleich wichtige nur angedeutet; das Material illustrierender Tatsachen gesammelt, aber kaum gruppiert, geschweige verarbeitet; am Schluß der Kapitel, unter dem Drang zum nächsten zu kommen, oft nur ein paar

\footnotetext{
W. Schluchter $(\square)$

Max-Weber-Institut für Soziologie, Universität Heidelberg, Bergheimer Str. 58, 69115 Heidelberg, Deutschland

E-Mail: wolfgang.schluchter@mwi.uni-heidelberg.de
} 
abgerißne Sätze als Marksteine der hier unvollendet gelaßnen Entwicklung; endlich die bekannte, dem Verfasser selbst manchmal unleserliche Handschrift." (Engels 1983, S. 7) Kurz: Der Herausgeber sah sich der schwierigen Aufgabe gegenüber, den Text erst einmal lesbar zu machen. Er konnte ihn nicht einfach nur dokumentieren, er musste ihn auch interpretieren und ergänzen, musste sich nicht nur als Herausgeber, sondern in gewissem Sinn als Mitautor verstehen.

Nachdem Max Weber am 14. Juni 1920 in München gestorben war, fand man auch in seinem Nachlass eine Fülle von begonnenen und nicht abgeschlossenen Manuskripten, allerdings längst nicht so zahlreich wie bei Karl Marx. Aber wie auch bei diesem, schienen sie hauptsächlich zu einem seiner Hauptwerke zu gehören, zu Wirtschaft und Gesellschaft, womit er sich seit 1910 beschäftigte. Sie waren jedoch, anders als dies Engels von Marx' Manuskripten berichtet, keineswegs durchweg alle in einem vorläufigen Zustand. Allerdings: Die Abfolge der vorgefundenen Manuskripte schien nicht immer klar zu sein. Seit Weber die Arbeit an Wirtschaft und Gesellschaft begonnen hatte, waren 10 Jahre vergangen, wobei er während des Krieges, von 1914 bis 1918, nicht daran arbeitete. Dass er 1910 die Arbeit daran überhaupt in Angriff genommen hatte, war Folge seiner 1909 getroffenen Entscheidung, die NeuOrganisation eines Handbuchs der politischen Ökonomie zu übernehmen und sie redaktionell zu betreuen. Dieses Handbuch, beim Verlag J. C. B. Mohr (Paul Siebeck) in Tübingen erschienen, hatte bereits mehrere Auflagen erlebt, musste aber nach dem Tod des ursprünglichen Herausgebers Gustav von Schönberg neu konzipiert und auf den inzwischen erreichten Wissensstand gebracht werden. Diese Aufgabe übernahm Max Weber, wenn auch zunächst eher zögerlich. Schließlich entwarf er für dieses neue Handbuch im Jahre 1910 eine Gliederung (einen „Stoffverteilungsplan“), warb Mitarbeiter an und teilte sich selbst mehrere Beiträge zu, darunter einen mit dem Titel „Wirtschaft und Gesellschaft“. Dieser sollte ursprünglich drei Themenbereiche umfassen: Wirtschaft und Recht, Wirtschaft und soziale Gruppen sowie Wirtschaft und Kultur (Weber 2009, S. 145 f.).

Was für Karl Marx der Freund, war für Max Weber seine Frau. Marianne Weber galt als eine bedeutende Vertreterin der bürgerlichen Frauenbewegung und war sowohl wissenschaftlich wie politisch äußerst aktiv. Sie kannte Max Webers Absichten, konnte auch seine schwer lesbare Handschrift entziffern. Vieles des im Schreibtisch gefundenen Materials war allerdings bereits maschinenschriftlich verfasst. Ein Jahr vor seinem Tode hatte Max Weber mit der Veröffentlichung nicht nur von Wirtschaft und Gesellschaft, sondern auch der Gesammelten Aufsätze zur Religionssoziologie begonnen. Zum Zeitpunkt seines Todes waren dreieinhalb Kapitel von Wirtschaft und Gesellschaft und der Band 1 der Gesammelten Aufsätze zur Religionssoziologie im Druck. Für seine Sammlung religionssoziologischer Aufsätze, die vier Bände umfassen sollte, hatte Weber bereits 1919 eine Disposition veröffentlicht, für Wirtschaft und Gesellschaft fehlte sie. Das stellte Marianne Weber vor ein Problem, das Engels in dieser Art nicht hatte. Sie musste zwar nicht wie dieser in die Texte selbst eingreifen, wohl aber die im Druck befindlichen Texte mit den im Nachlass gefundenen Manuskripten zusammenbringen, ohne dafür ein Inhaltsverzeichnis letzter Hand zu besitzen. Die Lösung, die sie dafür wählte, führt uns zu den Voreditionen, die vorlagen, als die Arbeit an der Max Weber-Gesamtausgabe begann. 


\section{Die Ausgaben der Schriften Max Webers von 1920 bis 1976}

Die Geschichte der Voreditionen der Werke Max Webers lässt sich in zwei Phasen unterteilen: Die erste Phase bestimmte Marianne Weber, die zweite Johannes Winckelmann. Die erste Phase reicht von 1920 bis 1954, dem Todesjahr Marianne Webers, die zweite von 1954 bis 1985, dem Todesjahr Johannes Winckelmanns. Die beiden Phasen überschneiden sich, denn bereits 1951 war Johannes Winckelmann an der zweiten Auflage der ursprünglich von Marianne Weber herausgegebenen Gesammelten Aufsätze der Wissenschaftslehre beteiligt. 1956 erscheint dann Winckelmanns Fassung von Wirtschaft und Gesellschaft, die von Marianne Webers Fassung abweicht. Dazu gleich mehr.

Zunächst zur ersten Phase. Sie beginnt bereits in München, unmittelbar nach Max Webers Tod, setzt sich dann in Heidelberg fort, wohin Marianne Weber zurückgekehrt war. Die wichtigste Aufgabe für Marianne Weber bestand zunächst darin, die im Druck befindlichen Kapitel von Wirtschaft und Gesellschaft aus dem Nachlass zu ergänzen. Es handelte sich um insgesamt 19 Texte, von denen manche intern gegliedert, andere nicht abgeschlossen, wieder andere noch handschriftlich überliefert waren. Von diesen Texten nahm sie an, sie seien nahezu alle von Max Weber für sein Buch mit dem Titel Wirtschaft und Gesellschaft bestimmt gewesen. Dieses Buch, so hatte es Max Weber mit seinem Verleger verabredet, sollte gemäß dem Arbeitsfortschritt des Autors in Lieferungen veröffentlicht werden, in Broschüren, die nach Abschluss des Projekts zusammengebunden und so zu einem Buch vereinigt werden sollten. Marianne Weber behandelte die im Druck befindlichen Kapitel als die erste Lieferung. Ihr sollten also weitere Lieferungen folgen, die aus den nachgelassenen Manuskripten bestritten werden mussten. So kam es schließlich zu drei weiteren Lieferungen. Die erste Auflage von Wirtschaft und Gesellschaft ist also aus vier Lieferungen bzw. Broschüren zusammengesetzt. In der Zeit von März 1921 bis Juli 1922 gab es insgesamt nicht weniger als sieben Versuche, die nachgelassenen Manuskripte in eine sachlich begründete Reihenfolge zu bringen. ${ }^{1}$ Das kann hier nicht weiter verfolgt werden, zeigt aber, wie kompliziert die Anordnung war. Es erklärt auch, weshalb wir es bei Wirtschaft und Gesellschaft mit einer Merkwürdigkeit zu tun haben: Mit Ausnahme der dritten Auflage von 1947, die eigentlich keine eigene Auflage ist, sondern nur ein Nachdruck der zweiten Auflage von 1925, ist keine der Auflagen von Wirtschaft und Gesellschaft $(1922,1925,1956,1972)$ mit einer anderen textgleich. ${ }^{2}$

Die Anordnung komplizierte sich vor allem deshalb, weil Marianne Weber das bereits Gedruckte und das im Schreibtisch Gefundene als eine Einheit betrachtete, als Beiträge zu einem Buch in mehreren Teilen. Sie entschied sich für drei Teile, wobei sie allerdings zwischen dem ersten Teil, den Max Weber selbst noch zum Druck gegeben und korrigiert hatte, und den beiden anderen Teilen, die sie aus den im Schreibtisch gefundenen Manuskripten zusammensetzte, einen wichtigen Unter-

\footnotetext{
1 Vgl. dazu die Übersicht in Weber 2009, S. 130f. Marianne Weber wurde dabei von Melchior Palyi unterstützt.

2 Von der fünften Auflage, die von Johannes Winckelmann 1972 herausgegeben wurde, gab es sieben Nachdrucke.
} 
schied sah. Sie hatte nämlich den (richtigen) Eindruck, dass diese Texte sich im Duktus unterschieden. Um diesem Eindruck Rechnung zu tragen, bezeichnete sie den ersten Teil als Max Webers abstrakte (auch systematische), die beiden anderen Teile aber als Webers konkrete Soziologie (auch als ,schildernde Darstellung soziologischer Erscheinungen“; Weber 1972, S. XXXII). Sie beließ aber das Ganze in dem Rahmen, in dem es ursprünglich gestanden hatte, dem Handbuch der politischen Ökonomie, welches man 1914 aus pragmatischen Gründen in Grundriß der Sozialökonomik umbenannt hatte. Dennoch führte Marianne Webers Edition zu der Vorstellung, es gebe ein von Max Weber verfasstes Buch in drei Teilen unter dem Titel Wirtschaft und Gesellschaft, welches zudem sein unvollendetes Hauptwerk sei.

Dass Max Weber ein Buch schreiben wollte, steht außer Frage, nur nicht unter diesem Titel und nicht ohne gründliche Überarbeitung der im Schreibtisch gefundenen Manuskripte. Sein Titel sollte Soziologie lauten, und die Art der Überarbeitung kann man besonders gut an den Texten studieren, die doppelt überliefert sind. Das sind die Grundbegriffe, die Herrschaftssoziologie und die Ausführungen über Klassen und Stände. Für diese gibt es jeweils eine Vorkriegs- und eine Nachkriegsfassung. An ihnen kann man leicht erkennen, dass die Nachkriegsfassung die Vorkriegsfassung nicht ergänzen, sondern vielmehr ersetzen sollte. Max Weber hatte 1919 begonnen, das dicke alte Manuskript, wie er sagte, von Grund auf umzugestalten. Es diente ihm für die Nachkriegsfassung nur noch als Material.

Marianne Weber sah in der Veröffentlichung von Wirtschaft und Gesellschaft sowie der Gesammelten Aufsätze zur Religionssoziologie die vorrangigen Probleme, die zunächst gelöst werden mussten. Aber damit endete ihre Editionstätigkeit nicht. Man muss sich vor Augen halten, dass Max Webers umfangreiches Werk im Jahre 1920/21 dem größeren Publikum noch weitgehend unbekannt war. Viele seiner Beiträge steckten verstreut in Zeitschriften oder in Sammelwerken, waren nicht in Buchform präsent. Manches davon hatte zwar ein größeres Lesepublikum erreicht, wie etwa die zweiteilige Aufsatzfolge "Die protestantische Ethik und der ,Geist" des Kapitalismus“, die 1904 bzw. 1905 im Archiv für Sozialwissenschaft und Sozialpolitik erschienen war, in einer Zeitschrift, die Weber mit herausgab und die er regelmäßig mit eigenen Beiträgen belieferte. Doch neben der Veröffentlichung des Buches Wirtschaft und Gesellschaft und neben der Fortführung der Gesammelten Aufsätze zur Religionssoziologie um zwei weitere Bände - der erste Band war noch von Max Weber selbst zum Druck gegeben und korrigiert worden - war auch eine Sammlung der verstreuten anderen Schriften Max Webers ein Desiderat. Marianne Weber erfüllte es in Gestalt von insgesamt vier Bänden Gesammelter Aufsätze zur Politik, zur Wissenschaftslehre, zur Wirtschafts- und Sozialgeschichte sowie zur Soziologie und Sozialpolitik. Zusammen mit den Bänden zur Religionssoziologie - das überlieferte Material reichte nur für insgesamt drei Bände - ergaben sich daraus sieben Bände Gesammelte Aufsätze. Damit war das Werk Max Webers zum ersten Mal in seiner Spannweite und Vielgestaltigkeit sichtbar gemacht.

Doch Marianne Weber ließ es bei der Editionstätigkeit nicht bewenden. Sie schrieb auch die erste und lange Zeit maßgebende Weber-Biographie. In ihrem Buch Max Weber. Ein Lebensbild, 1926 erschienen, suchte sie Werkgeschichte und persönliche Biographie nichtreduktionistisch miteinander zu verknüpfen, ein trotz 
manch zeitbedingter Beschränkung heute immer noch lesenswertes Buch (Weber 1926).

Marianne Weber machte also nicht nur das Werk Max Webers zum ersten Mal einer bereiteren Öffentlichkeit zugänglich, sie bestimmte durch ihre Editionsentscheidungen auch die Rezeptionsgeschichte. Dies gilt insbesondere für die Vorstellung, dass Max Webers Hauptwerk Wirtschaft und Gesellschaft heiße und ein Buch in mehreren Teilen sei. Als nach Marianne Webers Tod Johannes Winckelmann zu einer Art Lordsiegelbewahrer des Weber'schen Werkes aufstieg, folgte er dieser Entscheidung, verwirklichte sie aber in gänzlich neuer Weise. Er machte aus der Dreiteilungsthese von Marianne Weber eine Zweiteilungsthese, indem er die Begriffstypologie von der Darstellung soziologischer Zusammenhänge und Entwicklungen trennte. Die Begriffstypologie sah er in Webers Nachkriegstext verwirklicht, den dieser noch zum Druck gegeben hatte, die Darstellung soziologischer Zusammenhänge und Entwicklungen aber in den Vorkriegstexten, die Weber bei Ausbruch des Krieges in die Schublade gelegt hatte und die Marianne Weber nach seinem Tod dort fand. Winckelmann vertrat die These, diese Manuskripte bildeten eine nahezu vollendete Einheit, die man nur richtig rekonstruieren müsse. Für diese Rekonstruktion müsse die von Max Weber im Jahre 1914 veröffentlichte Gliederung seines Hauptwerkes der Maßstab sein. Tatsächlich hatte Max Weber in dem inzwischen in Grundriß der Sozialökonomik umbenannten Sammelwerk kurz vor Ausbruch des Krieges eine Gliederung veröffentlicht, die er aber interessanterweise unter einen neuen Titel stellte: statt „Wirtschaft und Gesellschaft“ wie 1910 heißt er jetzt „Die Wirtschaft und die gesellschaftlichen Ordnungen und Mächte“. ,Wirtschaft und Gesellschaft" war zum Titel einer Abteilung des Sammelwerks geworden, für die außer Webers Beitrag ein zweiter, von einem anderen Autor geschriebener Beitrag vorgesehen war (Abteilung III: C. Wirtschaft und Gesellschaft. I. Die Wirtschaft und die gesellschaftlichen Ordnungen und Mächte; II. Entwicklungsgang der wirtschaftsund sozialpolitischen Systeme und Ideale). ${ }^{3}$ Ausgehend von der Annahme, Webers Beitrag unter I. habe bei Ausbruch des Krieges kurz vor seiner Vollendung gestanden, legte Winckelmann im Jahre 1956 seine Version des Buches unter dem Titel Wirtschaft und Gesellschaft als 4., neu herausgegebene Auflage vor. Er löste das Buch zudem aus dem Rahmen des Grundrisses der Sozialökonomik, in dem es ursprünglich gestanden und in dem es Marianne Weber belassen hatte, und er erfand stattdessen den Untertitel „Grundriß der verstehenden Soziologie“.

Es ist klar, dass Winckelmann damit die drei von Marianne Weber herausgegebenen Auflagen des Buches für obsolet erklären musste. Seine Polemik gegen seine Vorgängerin war scharf. Er warf ihr vor, nicht zu viel, sondern zu wenig interpretiert zu haben. Sie habe den inneren Zusammenhang von Webers Hauptwerk übersehen. Er stellte Texte um, veränderte interne Verweise, um die angebliche Konsistenz der Vorkriegsmanuskripte sichtbar zu machen. Er verschwieg nicht, dass es auch Lücken gab. Teilweise schloss er sie dadurch, dass er Texte erzeugte, etwa eine „Staatssoziologie“, zusammengestellt aus anderen Texten, die er aber nach heftiger Kritik später wieder aus Wirtschaft und Gesellschaft entfernte (siehe Weber 1956). Streng

\footnotetext{
3 Weber 2009, S. 168 f. Der Autor des zweiten Beitrages war Eugen von Philippovich.
} 
genommen hat er mit seiner Edition aber den von Marianne Weber eingeschlagenen Weg nicht verlassen. Im Gegenteil: Er hat ihn perfektioniert.

Johannes Winckelmann, von Haus aus Jurist und in seinem beruflichen Leben außerhalb der Universität tätig, fühlte sich als ein Schüler Max Webers, obgleich er nie bei ihm studiert hatte. Er sah in ihm einen demokratischen Hochschullehrer, der in seinen wissenschaftlichen und politischen Positionen seinen eigenen entsprach. Er ließ sich insbesondere von Webers herrschafts-, rechts- und verwaltungssoziologischen Analysen leiten und sah in ihm einen Kämpfer für eine Demokratie, wie sie ihm selbst auch für die Bundesrepublik Deutschland vorschwebte (siehe v.a. Winckelmann 1952). Winckelmann wollte Webers Werk nicht nur erhalten und möglichst konsistent formulieren, sondern es auch gegen Angriffe von rechts wie von links verteidigen. Man kann ohne Einschränkung sagen: Seit seiner Pensionierung stellte er seine ganze Arbeitskraft in den Dienst dieses Werkes. Er edierte es nicht nur, er interpretierte und kommentierte es auch. Der fünften Auflage von Wirtschaft und Gesellschaft, gegenüber der vierten in der Textzusammenstellung abermals verändert, gab er einen Kommentarband bei, mit dem zum ersten Mal der Versuch gemacht wurde, die weitgehend ohne Nachweise überlieferten Texte durch Literaturhinweise und Sachkommentare dem Leser zu erschließen. Das war auch für die Max WeberGesamtausgabe Vorbild. Was nicht übernommen wurde, ist Winckelmanns Neigung, die Textgestalt dem Systematisierungsinteresse des Editors zu unterwerfen und, statt zu dokumentieren, zu interpretieren. Das hat denn auch gewisse Spannungen im Herausgeberkreis der Max Weber-Gesamtausgabe zur Folge gehabt.

\section{Der Herausgeberkreis der Max Weber-Gesamtausgabe}

Der Plan, eine Max Weber-Gesamtausgabe zu beginnen, entstand in den 70er-Jahren des letzten Jahrhunderts. Die geistige Situation der Zeit schien dafür reif. Trotz der Editionen von Marianne Weber und Johannes Winckelmann hatte man das Werk Max Webers in Deutschland bis dahin eher selektiv wahrgenommen. Ein Indikator ist der Soziologentag 1964 in Heidelberg. Er war dem Thema „Max Weber und die Soziologie heute“ gewidmet (Stammer 1964). Sein Verlauf machte deutlich, dass Max Weber von ausländischen Wissenschaftlern umfassender rezipiert wurde und bei ihnen auch anerkannter war als im eigenen Land.

Hinzu kam das Abebben einer marxistischen Welle, die in den 1960er-Jahren das intellektuelle Leben im Lande bestimmte. Damit gelangten Themen wie Kapitalismus und Demokratie auf die Agenda. Dazu konnte man im Werk Max Webers viel Weiterführendes finden. Vor allem aber trat der Weber der Religionssoziologie immer stärker in den Mittelpunkt der Diskussion, und zwar nicht so sehr der Weber der „Protestantischen Ethik“ als vielmehr der Weber der „Wirtschaftsethik der Weltreligionen“, der eine vergleichende Forschung zu universalgeschichtlichen Problemen wie der „Rationalisierung“ betrieben hatte. Das weitete den Blick über die Gegenwart hinaus. Dabei entstand auch eine Debatte darüber, ob Wirtschaft und Gesellschaft tatsächlich als Max Webers Hauptwerk gelten könne oder ob nicht die Religionssoziologie diesen Titel verdiene (siehe Tenbruck 1975 und die weiteren Literaturhinweise in Schluchter 1988, S. 557 f.). Systematische und werkgeschichtli- 
che Fragen verknüpften sich. Konservative und liberale Kreise in der Bundesrepublik Deutschland schienen sich einig: Eine Gesamtausgabe der Werke eines der größten Sozialwissenschaftler des 20. Jahrhunderts war so etwas wie eine bundesrepublikanische Pflicht.

Wie der Kreis der fünf Herausgeber letztlich zusammenkam, ist aus der Erinnerung nicht leicht zu sagen. Jedenfalls ging die Initiative, ihn zu bilden, bereits 1973 von Horst Baier, Wolfgang J. Mommsen und Johannes Winckelmann aus (Lepsius 2016, S. 275). Sie hatten unterschiedliche Motive und unterschiedliche Kompetenzen. Winckelmann war seit 1954 Honorarprofessor an der Universität München und Leiter des Max Weber-Archivs sowie durch seine Herausgebertätigkeit eng mit dem Verlag J. C. B. Mohr (Paul Siebeck) in Tübingen verbunden. Er schien durch sein bisheriges Wirken für eine solche Aufgabe geradezu prädestiniert. Host Baier, von Haus aus Mediziner und Soziologe, hatte in Frankfurt a. M. den Lehrstuhl von Theodor W. Adorno übernommen und war dabei ins Zentrum der universitären politischen Auseinandersetzungen im Gefolge der Studentenbewegung geraten. Er hatte sich mit einer Arbeit über das Verhältnis von Heinrich Rickert und Max Weber habilitiert und sah Weber wohl als eine Alternative zu den marxistischen Strömungen. Wolfgang J. Mommsen, Historiker an der Universität Düsseldorf, hatte mit seiner Dissertation Max Weber und die deutsche Politik Furore gemacht (Mommsen 1974) und bei seinen Recherchen viele der zuvor unbekannten Quellen des Weber'schen Werkes erschlossen. Er war also nicht nur vertraut mit Weber, sondern auch mit der Methode der historischen Kritik. Zwischen Winckelmanns und Mommsens Auffassung, was eine Werk-Edition zu sein habe, gab es deshalb von Beginn an eine Spannung. Beide suchten M. Rainer Lepsius, Soziologe an der Universität Mannheim, gewissermaßen als Vermittler zu gewinnen. Er war seit langer Zeit mit beiden bekannt. Dieser wiederum holte Wolfgang Schluchter, Soziologe zunächst an der Universität Düsseldorf, dann an der Universität Heidelberg, also mich, in den Kreis, der ich gerade begonnen hatte, mich mit Teilen des Weber'schen Werkes systematisch zu beschäftigen, insbesondere mit der Frage, wie es zu anderen bedeutenden Theorien in der Soziologie stand (Schluchter 1972).

Der Herausgeberkreis, bestehend aus diesen fünf Personen, konstituierte sich förmlich im Sommer 1976 durch einen dreiseitigen Vertrag, der zwischen ihnen, der Bayerischen Akademie der Wissenschaften in München und dem Verlag J. C. B. Mohr (Paul Siebeck) in Tübingen geschlossen wurde. Darin ist die Bayerische Akademie der Wissenschaften als „Trägerin der historisch-kritischen Max WeberAusgabe" bezeichnet, die mit der Durchführung dieser Aufgabe die Kommission für Sozial- und Wirtschaftsgeschichte betraut. Der Verlag verpflichtet sich, die Herstellung der Ausgabe auf eigene Kosten zu übernehmen, und der Herausgeberkreis, die editorische Verantwortung für die Ausgabe zu tragen. Ihm oblag es, die Editionsprinzipien zu bestimmen, die Ausgabe zu gliedern und die Edition einzelner Bände an kompetente Personen zu vergeben. Etwa die Hälfte der Bände wurde von den Herausgebern und ihren Mitarbeitern und Mitarbeiterinnen selbst, der Rest von Externen ediert. 


\section{Die Edition}

Im Mai 1981 informierte der Herausgeberkreis die Öffentlichkeit über die Editionsprinzipien und den vorläufigen Aufbau der Ausgabe (Schluchter 1981). Er bekannte sich ausdrücklich zu einer dokumentierenden Edition, lehnte also eine interpretierende Edition ab. Es wurden drei Abteilungen eingerichtet: „I. Abteilung: Schriften und Reden; II. Abteilung: Briefe; III. Abteilung: Vorlesungsmanuskripte und Vorlesungsnachschriften“, später in „Vorlesungen und Vorlesungsnachschriften“ umbenannt. Während die Texte in den Abteilungen II und III rein chronologisch angeordnet werden sollten, wollte man für die Abteilung I thematische Einheiten bilden, innerhalb derer dann die Texte chronologisch zu ordnen waren. Es galt also eine Kombination von Pertinenz- und Chronologieprinzip. Der zu edierende Text musste der Text letzter Hand sein, Vorläufertexte waren mit Hilfe eines negativen Apparats auszuweisen. Das macht bei den Texten, wo Varianten vorliegen, die Textentwicklung transparent.

Jeder Text sollte textkritisch bearbeitet und kommentiert werden. Es gibt also für jeden Text zwei Apparate: einen für die Textkritik, einen für den Sachkommentar. Jedem Text war außerdem ein „Editorischer Bericht“ beizugeben, unterteilt in „Zur Entstehung“ und „Zur Überlieferung und Edition“. Jeder Band war zudem mit einer Einleitung des Editors und mit Registern zu versehen.

Es dauerte noch weitere drei Jahre, bis Anfang 1984 der erste Band dieser historisch-kritisch angelegten Gesamtausgabe erscheinen konnte. Der weitere Verlauf der Edition gestaltete sich mühsam, weil nahezu alle einzelnen Editionsprojekte länger dauerten als erwartet, mehrere Bandeditoren scheiterten und durch neue ersetzt werden mussten, auch die Finanzierung mitunter stockte, was mit der dezentralen Organisation der Editionsarbeiten zusammenhing (Düsseldorf, Heidelberg, München). Insgesamt dauerte die Edition länger, als die Lebenszeit der meisten Herausgeber reichte: Johannes Winckelmann starb 1985, Wolfgang J. Mommsen 2004 (der dann durch seinen Schüler Gangolf Hübinger, Historiker an der Universität Frankfurt an der Oder, ersetzt wurde), M. Rainer Lepsius 2014, Horst Baier 2017. Man ist in Abwandlung eines bekannten Spruches versucht zu sagen: Nicht die Revolution, sondern die Edition frisst ihre eigenen Kinder.

$\mathrm{Zu}$ Max Webers 100. Todestag am 14. Juni 2020 ist endlich der letzte noch fehlende Band der Max Weber-Gesamtausgabe erschienen. Es handelt sich zufällig um Webers Notizen zu seiner Vorlesung über Praktische Nationalökonomie. Damit hat die Max Weber-Gesamtausgabe folgendes Aussehen:

- Abteilung I: Schriften und Reden - 29 Bände

- Abteilung II: Briefe - 11 Bände

- Abteilung III: Vorlesungen und Vorlesungsnachschriften - 7 Bände

Die Gesamtausgabe umfasst also insgesamt 47 Titel, wobei mehrere Bände so umfangreich waren, dass sie geteilt werden mussten. Berücksichtigt man dies, so umfasst die Ausgabe zwar 47 Bandnummern, aber 54 Bände. 


\section{Wichtigste Ergebnisse}

Was sind nun die wichtigsten Ergebnisse dieser Edition? Ganz allgemein lässt sich sagen: Das Werk Max Webers ist nach 100 Jahren zum ersten Mal in seinem ganzen Umfang sichtbar, und die Max Weber-Gesamtausgabe bietet für die zukünftige Forschung eine verlässliche Textgrundlage, die nicht mehr ignoriert werden darf. Wer auch immer in Zukunft über und mit Max Weber arbeitet, wird auf diese Ausgabe nicht verzichten können. Auch wenn er andere Ausgaben benutzen möchte, so sollte er sie auf ihre Verlässlichkeit anhand der Gesamtausgabe überprüfen, was übrigens deshalb erleichtert wird, weil in der Gesamtausgabe die gängigen Voreditionen verzeichnet sind. Ich gehe im Einzelnen auf die drei Abteilungen ein.

\section{Abteilung I: Schriften und Reden}

Das wichtigste Ergebnis ist die Dekonstruktion von Wirtschaft und Gesellschaft. Es handelt sich hier nicht um ein Buch in Teilen, sondern um ein Projekt in mehreren Fassungen, und auch der ursprüngliche Titel „Wirtschaft und Gesellschaft“ stimmt bereits für die Fassung von 1914 nicht mehr. Denn dort ist „Wirtschaft und Gesellschaft" der Titel für eine ganze Abteilung des nun Grundriß der Sozialökonomik genannten Sammelwerks, die nicht nur Webers Beitrag umfassen sollte. Deshalb gab Weber seinem Beitrag auch den neuen Titel „Die Wirtschaft und die gesellschaftlichen Ordnungen und Mächte“, der übrigens sehr viel besser als der alte Titel zu dem hier vorgesehenen Inhalt passt.

Die Angelegenheit kompliziert sich nun aber noch dadurch, dass wir nicht einfach von einer Vorkriegs- und einer Nachkriegsfassung sprechen können, sondern bereits die Vorkriegsfassung zwei Fassungen enthält, die ineinander verschränkt sind. Es gibt eine erste Fassung, die 1910/11 entstanden ist, und eine zweite, die 1912 bis 1914 entsteht. Es ist aber nicht mehr möglich, diese beiden Fassungen auseinanderzuhalten. Es wäre eine Aufgabe der anschließenden Forschung, zu der die Gesamtausgabe einlädt, hier weitere Klärungen zu versuchen. Dafür stehen jetzt eine verlässliche Textgrundlage und übrigens auch ein Gesamtregister für alle Texte, die zum Projekt gehören, bereit (Weber 2015).

Nicht schwierig freilich ist es, die Vorkriegsfassung(en) von der Nachkriegsfassung zu unterscheiden. Man muss sich nur klarmachen, dass Weber zur Ausarbeitung der Nachkriegsfassung die Vorkriegsfassung nicht als Teil, sondern als Material benutzt. Er änderte dafür auch die Grundbegriffe. Die soziologischen Grundbegriffe von 1920 finden sich in den beiden Vorkriegsfassungen nicht. Der Vorläufer für die Grundbegriffe von 1920 ist der Aufsatz „Über einige Kategorien der verstehenden Soziologie", der selbst in sich zwei Textstufen aufweist und 1913 veröffentlicht wurde (Weber 2018). Die hier definierten „Kategorien“ finden sich in einigen der Vorkriegstexte wieder, aber eben nicht in allen. Schon dies macht deutlich, dass die Vorkriegsfassung nicht einheitlich ist.

An der Übersicht in Abbildung 1, rekonstruiert aus Dispositionen und Briefen, kann man sich die komplexe Überlieferungslage klarmachen.

Ein weiteres wichtiges Ergebnis ist die Erweiterung der politischen Schriften um die indirekt überlieferten Reden und Diskussionsbeiträge. Vor der Gesamtausgabe 


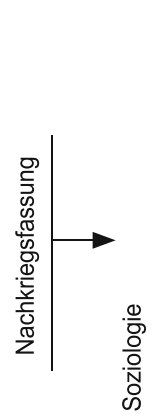

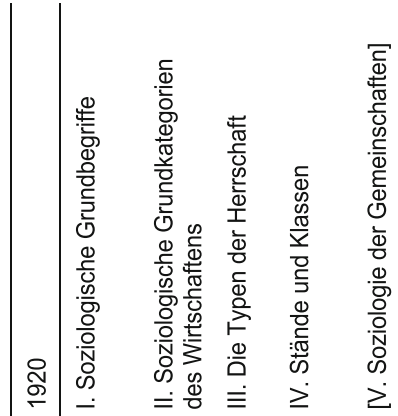

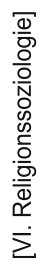
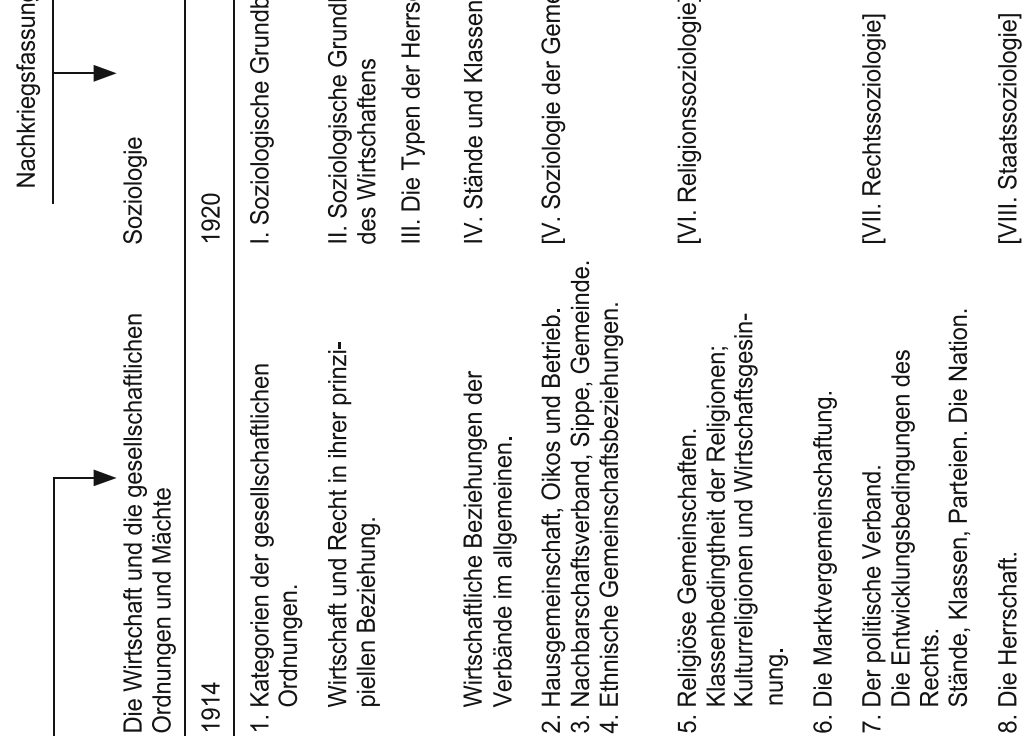

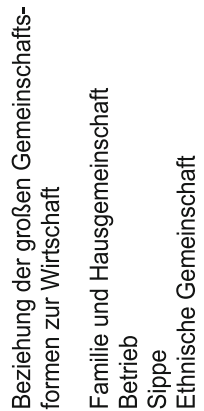

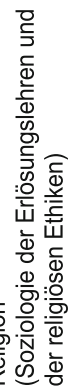

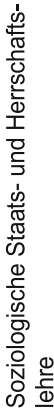
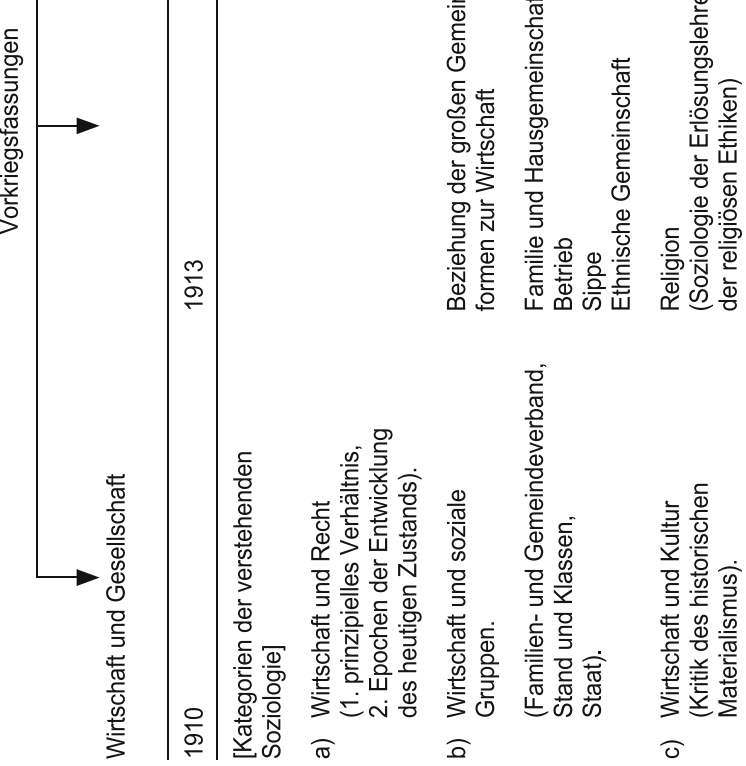

Abb. 1 Vergleich der Dispositionen von Max Webers Projekt „Wirtschaft und Gesellschaft“ aus den Jahren 1910 bis 1920 (Quelle: Weber 2013, S. 16) 
waren letztlich nur die von Weber zum Druck gegebenen Abhandlungen, Reden, Gutachten und sonstigen schriftlichen Stellungnahmen bekannt. Nun sind auch die indirekt in Form von Zeitungsberichten überlieferten Vorträge und Reden zugänglich, einschließlich der mitunterzeichneten Aufrufe und öffentlichen Erklärungen. Dies hat das Wissen um Max Webers politische Haltung am Ende des deutschen Kaiserreichs und im Übergang zur Weimarer Republik sehr vertieft.

\section{Abteilung II: Briefe}

Anders als die Schriften, von denen die meisten bekannt waren, sind die Briefe weitgehend eine neue Quelle für die Weber-Forschung. Die allermeisten der in den 11 Bänden chronologisch präsentierten Briefe und Karten waren bisher unbekannt. Viele Briefe werden mit einer editorischen Vorbemerkung eingeleitet und alle kommentiert, sodass trotz des Fehlens der Gegenbriefe der Zusammenhang, in dem das Geschriebene steht, jederzeit erkennbar ist.

Die Briefe und Karten sind zunächst einmal ein ausgezeichnetes Dokument der Kommunikationskultur des deutschen Bildungsbürgertums an der Wende vom 19. zum 20. Jahrhundert, als das Telefon den Brief als bevorzugtes Kommunikationsmittel noch nicht abgelöst hatte. Weber wurde von seinen Eltern bewusst mit dieser Briefkultur vertraut gemacht. Besonders die Jugendbriefe legen Zeugnis davon $\mathrm{ab}$, zeigen aber auch die Entwicklungsschwierigkeiten, die der junge Max Weber durchzustehen hatte. In den späteren Briefen erörtert er offen seine Lebensprobleme. Diese Zeugnisse sind unverzichtbar für jeden, der sich für Max Webers Lebenslauf interessiert.

Die Briefe haben aber auch eine große Bedeutung für die Interpretation seines wissenschaftlichen Werkes. Ich greife nur ein Beispiel heraus. In der Sekundärliteratur wird seit Langem über das Verhältnis zwischen Heinrich Rickert und Max Weber gestritten. Die einen sehen den Einfluss von Rickert auf Webers methodologische Position als zentral an, die anderen als peripher. In den Briefen finden sich nun viele Äußerungen, die diese Beziehung aus Max Webers Sicht erhellen, und zwar nicht nur in den Briefen an Rickert, sondern vor allem in den Briefen, in denen er sich gegenüber Dritten zu Rickert und seiner Bedeutung äußert.

Die Briefe und Karten geben auch Auskunft über Max Webers viele Reisen und die Beobachtungen, die er dabei machte. Besonders interessant sind seine Beobachtungen auf seiner nahezu dreimonatigen Reise durch die USA. In allen Reiseberichten besticht Weber durch scharfsinnige Betrachtungen zu den sozialen und kulturellen Verhältnissen im jeweiligen Land. Was er sieht, wird bei ihm gewissermaßen kategorial geformt.

Schließlich erfährt man in den Briefen viel über Webers Interessen an der Kunst, an Musik, Literatur, Malerei, Architektur, über die er teilweise geschrieben hat, aber noch ausführlicher schreiben wollte, es aber aus Zeitgründen nicht mehr konnte. Man sieht: Hier wartet auf die Forschung ein reiches Material. Man kann sich für die Zukunft keine Arbeit über Weber mehr vorstellen, die die Briefe-Edition nicht nutzte. Insofern enthält diese Abteilung einen Schatz, der noch gehoben werden muss und der nur in der Gesamtausgabe entdeckt werden kann. 
Abteilung III: Vorlesungen und Vorlesungsnachschriften

Wie im Fall der Briefe, bietet auch die Abteilung III überwiegend bisher unbekannte Texte. Das gilt insbesondere für die Vorlesungen aus der Zeit vor der Jahrhundertwende, vor Max Webers gesundheitlichem Zusammenbruch. Seit dem Sommersemester 1898 und vermehrt im Wintersemester 1898/99 hatte Weber Schwierigkeiten, seine Lehrverpflichtung zu erfüllen. In der Zeit danach erlebte er eine schwere gesundheitliche Krise, die ihn schließlich zum Rückzug von seiner Professur zwang. Man interpretierte diese Phase der krankheitsbedingten Arbeitsunfähigkeit häufig als einen tiefen Einschnitt, der Max Webers wissenschaftliche Ausrichtung verändert habe. Insbesondere in der Aufsatzfolge „Die protestantische Ethik und der ,Geist“ des Kapitalismus“, 1904 und 1905 erschienen, hat man einen solchen der Krankheitserfahrung geschuldeten Neuanfang gesehen. Nun besteht kein Zweifel: Webers Zusammenbruch von 1898/99 bedeutete zweifellos einen tiefen Einschnitt. Aber er hat sich nicht so stark, wie meist behauptet, auf das Werk ausgewirkt. Es lässt sich gerade an diesen frühen Vorlesungen zeigen, dass Weber bereits dort zum Beispiel das Thema der „Protestantischen Ethik“ behandelt und eine weit größere Kontinuität zwischen der Phase vor der Krankheit und der nach der Krankheit besteht, als die Meisten bisher vermutet haben.

\section{Die Rezeption der Gesamtausgabe}

Der lange Erscheinungszeitraum der Gesamtausgabe hat ihre Rezeption nicht unbedingt gefördert. Immerhin sind inzwischen einzelne Bände bereits in verschiedene Sprachen übersetzt worden. Da außerhalb des deutschsprachigen Raums Deutsch nur noch von wenigen gelesen wird, sind Übersetzungen unverzichtbar. Die Hoffnung allerdings ist, dass sie nun aus dem Original erfolgen und nicht aus einer anderen Übersetzung, etwa aus dem Englischen oder dem Japanischen.

Japan spielt für die Gesamtausgabe eine besondere Rolle. Als wir mit der Arbeit an der Edition begannen, fand dies in Japan eine große Resonanz. Seit den 20erJahren des letzten Jahrhunderts gibt es dort eine Weber-Rezeption wie in keinem anderen Lande. Über die Zeit produzierten die japanischen Gelehrten eine Sekundärliteratur über Weber in einer Menge, die fast so groß ist wie in der übrigen Welt. Auch die Exemplare der Gesamtausgabe gingen, kaum erschienen, außer nach Deutschland vor allem nach Japan. Lange Zeit übertraf der Absatz in Japan sogar den in unserem eigenen Land. Nur in diesen beiden Ländern gelang es dem Verlag, dreistellige Absatzzahlen zu erreichen. Kein anderes Land tat es Japan auch nur annähernd gleich. Nur noch in der Schweiz, in Österreich und in Italien kam der Verlag wenigstens auf zweistellige Absatzzahlen. Im Rest der Welt blieben sie einstellig, auch in den USA, oder gar bei null. Immerhin hat der Verlag sein Versprechen gehalten. Er produzierte diese auch drucktechnisch aufwendige Ausgabe ohne Zuschuss von außen, aus eigener Kraft.

Hätten wir in den 70er-Jahren des letzten Jahrhunderts gewusst, wie lange wir mit der Gesamtausgabe befasst sein würden, hätten wir das Unternehmen vermutlich nicht begonnen. Immerhin kann ich als letzter der ursprünglichen Herausgeber 
sagen: Es ist vollbracht. Die Gesamtausgabe wird sicherlich dazu beitragen, das Werk Max Webers lebendig zu halten. Man muss nur konsequent sein, d.h. sie nun auch noch vom analogen ins digitale Zeitalter überführen, sodass sie möglichst allen Interessierten zugänglich wird. Mit der ebook-Ausgabe, 2019 erschienen, wurde bereits ein Anfang gemacht.

Funding Open Access funding enabled and organized by Projekt DEAL.

Open Access Dieser Artikel wird unter der Creative Commons Namensnennung 4.0 International Lizenz veröffentlicht, welche die Nutzung, Vervielfältigung, Bearbeitung, Verbreitung und Wiedergabe in jeglichem Medium und Format erlaubt, sofern Sie den/die ursprünglichen Autor(en) und die Quelle ordnungsgemäß nennen, einen Link zur Creative Commons Lizenz beifügen und angeben, ob Änderungen vorgenommen wurden.

Die in diesem Artikel enthaltenen Bilder und sonstiges Drittmaterial unterliegen ebenfalls der genannten Creative Commons Lizenz, sofern sich aus der Abbildungslegende nichts anderes ergibt. Sofern das betreffende Material nicht unter der genannten Creative Commons Lizenz steht und die betreffende Handlung nicht nach gesetzlichen Vorschriften erlaubt ist, ist für die oben aufgeführten Weiterverwendungen des Materials die Einwilligung des jeweiligen Rechteinhabers einzuholen.

Weitere Details zur Lizenz entnehmen Sie bitte der Lizenzinformation auf http://creativecommons.org/ licenses/by/4.0/deed.de.

\section{Literatur}

Engels, F. (1983). [1885]. Vorwort. In K. Marx, Das Kapital. Kritik der politischen Ökonomie. Zweiter Band. K. Marx, F. Engels, Werke (MEW), Band 24 (S. 7-26). Berlin: Dietz.

Lepsius, M. R. (2016). Die Max Weber-Edition. In M. R. Lepsius, Max Weber und seine Kreise (S. 275-287). Tübingen: Mohr Siebeck.

Mommsen, W. J. (1974). [1959]. Max Weber und die deutsche Politik 1890-1920. 2., überarb. u. erw. Aufl. Tübingen: J. C. B. Mohr (Paul Siebeck).

Schluchter, W. (1972). Aspekte bürokratischer Herrschaft. Studien zur Interpretation der fortschreitenden Industriegesellschaft. München: List.

Schluchter, W. (1981). Einführung in die Max Weber-Gesamtausgabe. In Prospekt der Max Weber-Gesamtausgabe (S. 4-15). Tübingen: J. C. B. Mohr (Paul Siebeck).

Schluchter, W. (1988). Religion und Lebensführung. Band 2: Studien zu Max Webers Religions- und Herrschaftssoziologie. Frankfurt a. M.: Suhrkamp.

Stammer, O. (Hrsg.). (1964). Max Weber und die Soziologie heute. Verhandlungen des 15. Deutschen Soziologentags. Tübingen: J. C. B. Mohr (Paul Siebeck).

Tenbruck, F. H. (1975). Das Werk Max Webers. Kölner Zeitschrift für Soziologie und Sozialpsychologie, $17,663-702$.

Weber, M. [Marianne] (1926). Max Weber. Ein Lebensbild. Tübingen: J. C. B. Mohr (Paul Siebeck).

Weber, M. [Marianne] (1972). [1921]. Vorwort zur ersten Auflage. In M. Weber, Wirtschaft und Gesellschaft. Grundriß der verstehenden Soziologie. 5., rev. Aufl. (S. XXXII). Tübingen: J. C. B. Mohr (Paul Siebeck).

Weber, M. (1956). Staatssoziologie. Mit einer Einführung und Erläuterungen herausgegeben von Johannes Winckelmann. Berlin: Duncker \& Humblot.

Weber, M. (2009). Wirtschaft und Gesellschaft. Entstehungsgeschichte und Dokumente. Dargestellt und herausgegeben von Wolfgang Schluchter. Max Weber-Gesamtausgabe, I/24. Tübingen: J. C. B. Mohr (Paul Siebeck).

Weber, M. (2013). Wirtschaft und Gesellschaft. Soziologie. Unvollendet 1919-1920. Herausgegeben von Knut Borchardt, Edith Hanke und Wolfgang Schluchter. Max Weber-Gesamtausgabe, I/23. Tübingen: J.C. B. Mohr (Paul Siebeck).

Weber, M. (2015). Wirtschaft und Gesellschaft. Gesamtregister. Bearbeitet von Edith Hanke und Christoph Morlok. Max Weber-Gesamtausgabe, I/25. Tübingen: J. C. B. Mohr (Paul Siebeck). 
Weber, M. (2018). [1913]. Über einige Kategorien der verstehenden Soziologie. Max Weber-Gesamtausgabe, I/12 (S. 389-440). Tübingen: J.C. B. Mohr (Paul Siebeck).

Winckelmann, J. (1952). Legitimität und Legalität in Max Webers Herrschaftssoziologie. Mit einem Anhang: Max Weber: Die drei reinen Typen der legitimen Herrschaft. Tübingen: J. C. B. Mohr (Paul Siebeck). 